\title{
La liberté impossible et nécessaire
}

\author{
Pierre Verstraeten
}

I.

Deux citations pour commencer, d'abord «L'Entretien avec M. de Saci » dans les Pensées et Opuscules. Là, Pascal nous dit :

les deux plus célèbres défenseurs des deux plus célèbres sectes du monde et les seules conformes à la raison, puisqu'on ne peut suivre qu'une de ces deux routes, à savoir : ou qu'il a un Dieu, et alors il y place son souverain bien ; ou qu'il est incertain, et qu'alors le vrai bien l'est aussi, puisqu'il en est incapable (Pensées 159).

Pascal pointe ici le stoïcisme orgueilleux qui connaît trop bien la grandeur de l'homme, mais au point d'ignorer ses faiblesses, sa part d'ombre. À ce titre, Epictète n'a pas fait sa part à l'impuissance humaine. D'autre part Pascal parle d'une « superbe diabolique » (150) et d'un scepticisme aimable et subtil à la Montaigne, «doute qui doute de soi et ignorance qui s'ignore » (151). Il ignore la puissance de l'homme et ne prend en compte que sa fragilité. Ce dernier a bien connu la fragilité et l'inconséquence de la raison humaine. Seulement son scepticisme conduit à la lâcheté, faute d'avoir conscience de la grandeur de l'homme :

Humiliez-vous, raison impuissante; taisez-vous, nature imbécile : apprenez que l'homme passe infiniment l'homme, et entendez de votre maître votre condition véritable que vous ignorez. Écoutez Dieu (no. 434).

N'oublions pas que :

Les choses du monde les plus déraisonnables deviennent les plus raisonnables à cause du dérèglement des hommes. Qu'y a-t-il de moins raisonnable que de choisir, pour gouverner un État, le premier fils d'une reine? On ne choisit pas pour gouverner un vaisseau celui des voyageurs qui est de la meilleure maison. Cette loi serait ridicule et injuste; mais parce qu'ils le sont, et le seront toujours, elle devient raisonnable et juste, car qui choisira-t-on, le plus vertueux et le plus habile? Nous voilà incontinents aux mains, chacun prétend être ce plus vertueux 
et ce plus habile. Attachons donc cette qualité à quelque chose d'incontestable. C'est le fils aîné du roi; cela est net, il n'y a point de dispute. La raison ne peut mieux faire, car la guerre civile est le plus grand des maux (no. 320).

Bref : l'ordre social, du point de vue de l'habile (Pascal lui-même), est fondé sur des raisons qui ne sont pas celles que la société se représente à elle-même. L'ordre est imparfait, mais il est à la mesure de notre condition. À propos du stoïcisme et du scepticisme pascaliens Sartre serait passé du premier au second, du moins selon une certaine interprétation du sens de la liberté présenté dans les conclusions morales de L'etre et le néant, son ouvrage philosophique fondamental. Nous verrons aussi que ce qui apparaît comme le fin mot du sens et de l'usage de la liberté chez Sartre, ne serait à ses yeux qu'une position analogue à celle des « demi habiles » chez Pascal ; hypothèse utile pour saisir le fréquent contre-sens concernant ce passage de la conclusion de L'etre et le néant. C'est en échappant à ce contre-sens, à la suite de Christina Howells, que nous comprendrons le sens paradoxal de la liberté sartrienne.

La liberté chez Sartre ne devrait pas poser problème. Je veux dire que son affirmation en elle-même n'est pas paradoxale : c'est l'affirmation la plus récurrente de son œuvre, de sorte que si l'on connaît un tant soit peu l'auteur, son affirmation relève du leitmotiv. J'ajouterais, pour ceux qui en connaissent un peu plus sur l'histoire de la philosophie, que c'est un thème plus que classique de la philosophie occidentale depuis Descartes, Leibniz, Kant, Hegel et tout l'idéalisme allemand.

On se demandera alors où est la différence de ces systèmes si la liberté constitue le thème essentiel de chacun. En fait, cette différence, ces philosophies ne la détiennent pas en elles-mêmes, c'est-à-dire par la compréhension intrinsèque de la spontanéité de la liberté, de son noyau ontologique, mais par l'économie des systèmes de chacun. Au reste, pour parler généralement de cette tradition de la liberté en philosophie, tout penseur est nécessairement conscient de sa liberté dans l'exercice de sa pensée. Sinon que pourrait bien vouloir dire une 
activité philosophique (c'est-à-dire une création de la pensée), sans la conscience de la responsabilité de l'autonomie intellectuelle intégrale de son auteur ? Ce qui veut dire qu'une pensée non libre ne serait pas une pensée! Sartre nous l'affirme philosophiquement dès l' « Introduction » de L'etre et le néant non seulement de la pensée, mais aussi de l'existence elle-même :

Ou bien je ne suis pas passif en mon être, alors je deviens le fondement de mes affections même si tout d'abord je n'en ai pas été l'origine - ou bien je suis affecté de passivité jusque dans mon existence, mon être est un être reçu et alors tout tombe dans le néant (L'etre et le néant 25).

Comprenons ici : si pour une raison ou l'autre, je me trouve ou me sens offensé, en considérant d'un point de vue extérieur et objectif que l'offense est réelle et voulue, je suis libre par rapport à elle par la manière dont j'y réagis : colérique, indifférente, mortifiée, indignée, etc. Précisons que le fait même de se sentir offensé n'existe pas en soi, mais poursoi, c'est-à-dire en situation et par la manière d'y réagir. Et rien de plus vrai. À cet égard, on le sait, les seuils de susceptibilité en général sont très variables : il y a les écorchés vifs et il y a les peaux de rhinocéros, etc. Mais « pour ceux à qui on ne la fait pas » (entendons là les « malins » ou les fins psychologues, ou les « demi-habiles »), c'est justement cette manière qui ne dépend pas de la liberté mais de la constitution originaire (biologique, psychologique ou même sociale et idéologique) de chaque individu. À ce titre, l'offense serait bien l'effet d'un déterminisme puisque la manière d'y réagir serait prédéterminée.

On peut dire, dans cette hypothèse, que c'est à ce moment là que le second terme de l'alternative de Sartre tombe comme une sentence ou, mieux encore, comme une condamnation : «Ou bien je suis affecté de passivité jusque dans mon existence, mon être est un être reçu et alors tout tombe dans le néant. » Car c'est bien de cela dont il s'agit dans cette hypothèse : de la passivité idoine de l'existence. Mais dans ce cas on ne voit plus très bien ce que voudrait dire «exister. » Exister serait simplement «être » au sens des choses, des 
minéraux ou des végétaux : c'est-à-dire le produit d'un ensemble d'entrechoquements entre le monde et l'être considéré (chacune de nos consciences). Dès lors, de notre point de vue humain, convaincu du caractère exceptionnel de notre conscience, cette interprétation ferait de notre conscience une superstructure illusoire de notre toute puissance ; ce qui signifierait « tomber dans le néant, » dans l'inanité signifiante des croyances idéologico-culturelles les plus chevillées à notre esprit. Il s'agit ici du moment proprement spéculatif d'affirmation de la liberté - une preuve apagogique ou par l'absurde : si vous acceptez le conditionnement (psychanalytique, par exemple), alors vous aurez telle conséquence que vous ne pouvez que récuser horrifiés : l'homme cesserait d'être un homme, etc.

II.

Voici donc les faits conceptuels de la situation théorique présentée par Sartre. Il faut maintenant en tenter une analyse plus pratique pour mesurer l'éventuel sens de son usage. Il est certain d'abord que Sartre a nourri son affirmation de la liberté, pure spontanéité et contingence, d'un stoïcisme moral sans réserve durant ses premières années philosophiques et dont il s'est expliqué avec netteté dans les Carnets de la drôle de guerre. C'est ce que Pascal appelait un stoïcisme radical. Mais dans ces mêmes Carnets justement, Sartre s'en est détaché en arguant de son expérience concrète de l'histoire : cette guerre est à moi, je la suis dans la mesure où je ne peux pas ne pas l'assumer, c'est-à-dire d'une manière ou l'autre la vivre... soit comme déserteur, tireur au flanc, zélé météorologue, fraternisant les rapports entre tous ses acolytes ou au contraire radicalisant un antagonisme latent entre ces derniers, dans ce dernier cas l'antagonisme résulterait de la rancour d'être là ou d'un sentiment de supériorité du fait d'y être sans y être par son appartenance antérieure à une élite ... et par l'évasion symbolique constituée par son écriture des carnets. 
Bon, la guerre est ignoble, reconnaît-il, comme sans doute toute guerre, mais elle définit son-être-au-monde au présent. En la désertant n'aurait-il donc plus d'être-au-monde ? Non, il aurait un autre être-au-monde, un être-au-monde pour et dans le néant du futur. Dès lors, qui sait ? Ne perdrait-il pas en même temps tous les avantages et les charmes qu'il peut tirer de sa situation présente ? Sa correspondance suivie avec ses amies, son écriture des carnets se nourrissant de ce présent, et des découvertes philosophiques qu'il y fait, etc.

Je ne peux donc nier mon enracinement présent en me réfugiant dans la place forte de ma conscience et le néant du futur (de ma liberté), faute de ne plus savoir ce que je nie au présent par le futur, donc incapable de le nier, et dans l'incapacité de jouir de tous les avantages développés par la situation dans son actualité présente quels qu'en soient les inconvénients, comme cette jeune fille boiteuse à laquelle l'amoureux, vivant son amour au présent et face à la réalité de son objet, prête un charme capricieux à la démarche. Le stoïcien orgueilleux s'en gausserait considérant que l'amoureux se paye de mots pour sublimer son désir et le cristalliser en amour. Mais précisément pour Sartre il faut être amoureux pour saisir le mystère capricieux de la démarche de la belle, s'en moquer n'a aucun sens. Car on sait bien que fait défaut au moqueur le travail du désir, de la séduction et de l'amour, bref fait défaut le tout du rapport à l'être actuel, pour être en mesure de même savoir ce qu'il prétend nier par sa moquerie : comment le stoïque pourrait-il se réfugier dans sa liberté intérieure s'il n'est plus soutenu par la relation extérieure à ce dont il veut se défendre ? Si c'est le cas, s'il vit en partie double, comment peut-il soutenir la contradiction de vivre une chose et de dire le contraire, aimer et disqualifier l'objet de son amour par dépit, par ressentiment ou réactivité ?

Imaginons maintenant un stoïcien - prétendument à la hauteur exclusive de sa liberté - abandonné par sa belle. Qu'à cela ne tienne, elle n'avait pas les qualités dont il l'auréolait : 
cette belle jeune fille élancée devient à ses yeux un grand cheval. Sartre appelle ça la sournoiserie du stoïcisme qui met en place une défense rhétorique face à une expérience vécue pénible. L'homme authentique doit au contraire se plaindre et gémir, critiquer, implorer même, mais sans renier le désir et l'amour qu'il portait à son aimée. Ce sera vivre pleinement la condition humaine : c'est-à-dire conserver l'idéalité de ses sentiments au sein de la détresse psychique de l'abandon subi. Fi des faux-fuyants du stoïque. On ne peut couper l'homme en deux.

Ceci est possiblement difficile à comprendre puisque Sartre semble faire une différence entre ce qui affecte le pathos de l'homme (la tristesse pathétique d'être abandonné) et ce qu'il nourrissait d'élans proprement idéaux et spirituels dans son amour. Mais pour Sartre les deux dimensions (affectives et spirituelles) sont intimement liées. Elles le sont même forcément; car il n'y aurait pas détresse sans amour, et pas d'amour sans la composante psychique et physique de ce sentiment, sans entre autres la jubilation accompagnant au niveau du corps ce sentiment idéal ; jubilation qui se transforme en détresse s'il y a perte de l'autre. C'est ce que Sartre appelle la Transcendance et la Facticité, entre lesquels « un équilibre doit pouvoir s'établir. »

\section{III.}

Dans les Carnets Sartre revient sur son stoïcisme ou revient de son stoïcisme orgueilleux à la considération de ce qui pour Pascal sera le scepticisme fatigué ; en l'occurrence Montaigne qui considère l'impossibilité de s'en tenir à seulement un des deux termes alternatifs puisque l'homme est les deux : à savoir grandeur et faiblesse, universalité et vanité, ou, chez Sartre, transcendance et facticité, liberté et opacité des corps. À ce propos et semblablement Kant (dans La Religion dans les limites de la simple raison) critiquait déjà le 
stoïcisme en dénonçant cette doctrine comme une morale de et pour «Saint »; alors que l'homme trouve sa grandeur ou sa spécificité dans le fait d'être les deux : idéalité et pathos sensible. Mais alors que Kant, comme Sartre, considère qu'une synthèse doit ou peut être soutenue entre les deux dimensions (fragilité et puissance), les demi habiles (eux, qui apprécient toujours raisonnablement sinon rationnellement la situation, conscients en l'occurrence des deux dimensions de l'homme, observant sa grandeur et sa faiblesse, ou l'élite éclairée et le peuple) semblent conclure à l'équivalence de l'un ou l'autre engagement, donc adoptent le scepticisme fatigué au nom de l'unicité de la synthèse de chacun des pôles : facticité et transcendance, vus chacun comme des éléments homogènes. Il s'agit donc aussi d'une synthèse, mais autrement répartie : non plus entre les deux pôles antagonistes (faiblesse et grandeur), mais la synthèse de soi à soi, de soi dans sa faiblesse ou de soi dans sa grandeur. C'est que, comme toute option adoptée, elle manifestera forcément une dimension de facticité (la couardise physique du faible en général) en même temps que la dimension de transcendance de ce choix (la justification plaidée de cette lâcheté), et semblablement pour la grandeur (la témérité au niveau de sa facticité, et l'ambition affichée au niveau de son courage pour le fort).

Mais alors en quoi cette synthèse peut-elle être rangée - en fonction de ce que va en dire Sartre - comme relevant d'une attitude de demi habiles, et pourquoi ? En quoi, tout d'abord ? Parce que cette option de synthèse, pour originale qu'elle soit par rapport à Kant et toute la tradition chrétienne (tout en semblant également refléter le point de vue de Sartre), relève précisément d'une décision caractéristique des demi habiles de Pascal. Ce qui correspond au «bon sens » s'alignant sur la rationalité objective de la situation, au sein de laquelle la décision doit être prise, en l'occurrence: ne pas séparer ou hiérarchiser l'antagonisme entre facticité et transcendance, mais, bien plutôt, montrer l'unicité de la 
double dimension de chaque option permettant de les renvoyer dos-à-dos, chacune bénéficiant de l'autorité de son choix libre, librement lâche ou librement courageux, de part en part, synthétisant dès lors par leur liberté leur transcendance et leur facticité. Cette position privilégie apparemment l'interprétation kantienne du critère exclusif de la loi morale, ou encore de son formalisme. Quelle autre universalité concevable, en effet, que celle de la liberté ? Et en quoi s'agit-il dès lors d'une position assimilable à celle des demi habiles ?

En ce que dans ce cas la liberté devient le mot fétiche pour cimenter l'option adoptée. Elle est la justification de chaque revendication. C'est en ce sens qu'on connaît la phrase, correspondant au scepticisme fatigué, par laquelle Sartre désigne cette attitude. Ils en relèvent dans la mesure où les protagonistes se donnent respectivement la vocation de faire ce qu'ils font pour atteindre à la synthèse (consciente nécessairement) du néant de la conscience et de la plénitude de l'être. Que ce soit (par) l'action du conducteur de peuple brassant et agitant la multiplicité des masses pour les faire accéder à l'absoluité d'une fusion, ou que ce soit (par) une sorte de mimétisme subi à l'égard de l'être, de l'être des choses ou de l'être des autres, comme un ivrogne hébété et fasciné par sa propre passivité. Il faut bien comprendre que leur équivalence en liberté revient au même, et à ce titre (la conscience de soi de son choix) le pire peut même l'emporter sur ce qui apparaît le meilleur : tout se trame dans le rapport de soi à soi de la liberté de chacun. Indécidable dès lors. Ce qui explique l'indifférence du sceptique sur les contenus de l'engagement.

On voit la justification pour chacune des perspectives : atteindre la synthèse de l'être et du néant pour le conducteur de peuple, en tant que conducteur du peuple, ou la synthèse de l'être et du néant pour l'ivrogne solitaire, en tant qu'ivrogne solitaire. Ce qui est tout le contraire d'une synthèse, forcément hiérarchisée, dans la juxtaposition de la facticité de l'hébétude alcoolique et de la transcendance idéologico-politique et militante ; mais, bien 
plutôt, la synthèse de chacune des deux positions en et par elles-mêmes : être des essences pures du Révolutionnaire ou de l'Ivrogne solitaire, à l'instar de l'Essence pure de quoi que ce soit revendiquée déjà en général par Platon : c'est-à-dire une mère rien d'autre que mère, une essence de mère, femme-mère qui ne sera ni fille elle-même, ni tante, ni cousine, ni maîtresse, mais Mère. Ainsi pour la synthèse projetée par les demi habiles : un pur Leader, auréolé de la seule gloire de son image passée et future en même temps que de la simultanéité de son action militante, ou un pur Ivrogne imbibé sinon englouti dans le nirvana de son hébétude et revendiquant son destin d'alcoolique. « Comment s'empêcher d'éprouver une insupportable pitié pour les pierres, une identification pétrifiante » dira Deleuze (Logique du Sens 181), à propos du héros alcoolique du roman de Malcolm Lowry Sous le Volcan. Ainsi, dans les deux cas, nous voyons la synthèse entre la transcendance de l'un et de l'autre (l'idéologie de chacun) et la facticité (le corps engagé de chacun) et leur équivalence du point de vue du critère de la liberté. Liberté des demi habiles en tant que sceptiques fatigués. Reste à savoir pourquoi les tenants de cette attitude philosophique se trouvent disqualifiés à l'instar des demi habiles de Pascal, dès lors forcément au nom d'une position d'habile?

Nous aurions pu croire avoir atteint là le sens ultime de l'idéal pratique de la liberté sartrienne. Mais non. Il ne faut pas oublier que cette dernière visée par l'un ou l'autre des protagonistes mis en scène dans L'etre et le néant relève de ce que, comme nous l'avons dit, Christina Howells n'hésite pas à assimiler aux demi habiles de Pascal. Il s'agira alors de voir où se place donc l'insuffisance de cette visée de la synthèse idéale d'être intégralement ce qu'on fait, corps et âme : leader des masses ou alcoolique solitaire ? Quelle était donc la formule apparemment positive de la thèse qui vient d'être exposée par Sartre ? Je répète et cite Sartre :

Si l'une de ces [deux] activités l'emporte sur l'autre, ce ne sera pas à cause de son but réel, mais à cause du degré de conscience qu'elle possède de son but idéal ; et dans ce 
cas, il arrivera que le quiétisme de l'ivrogne solitaire l'emportera sur l'agitation vaine du conducteur de peuples (L'etre et le néant 722).

J'ai cru longtemps avoir là le dernier mot de la perspective morale de Sartre dans L'etre et le néant. L'essentiel apparaissant être le degré de lucidité de ce qu'on fait, donc «la mise au poste de commandement de la liberté. » Non pas le contenu de chacun des actes, mais le degré de lucidité quant à la libre responsabilité de l'engagement à son propos. Eh bien, non ! La Raison ne peut être le dernier mot de la liberté à l'instar de demi habiles. Les habiles, pour autant qu'il y en ait, se situent au-delà de la Raison, ou encore s'alignent sur la formule de Pascal : l'existence [le cœur] possède ses raisons que la raison ne connaît pas. Autrement dit, il y a une dimension de la pensée, comme chez Pascal ou Kierkegaard, qui en appelle à la religion pour rendre compte de cet au-delà. Comme le font les habiles de Pascal qui adoptent le point de vue populaire, une sorte de foi aveugle dans la tradition, mais en sachant pourquoi. Heidegger, encore, dira que « le sens de la vérité c'est la liberté. »

En fait, la justification apportée par les habiles trouvera la genèse de son explication dans l'origine de la division due au péché originel, à savoir le partage de l'homme entre les forces du mal et celles du bien. Dès lors la réconciliation entre les hommes ou leur salut ne pourra venir que de la croyance à la transcendance religieuse, dont la raison ne peut rien dire ou face à quoi elle est impuissante. En effet croire en Dieu est absurde. Puisqu'il est caché et abscons pour Pascal et qu'il est paradoxal d'y croire comme Job ou Abraham pour Kierkegaard. Mais ceci ne constitue certes pas la justification de l'au-delà de la raison pour Sartre, on l'imagine aisément. Alors pourquoi est-il d'accord avec la formule de Heidegger selon laquelle l'essence de la vérité c'est la liberté ?

Comprenons-le par lui-même en nous servant des deux illustrations sartriennes d'éventuels demi habiles. Ils assujettissent précisément leur liberté à la Raison, ou à l'Idée de leur essence respective, c'est-à-dire à l'adéquation poursuivie entre ce qu'ils font et ce qu'ils 
sont. Le tort de cette séduisante thèse, c'est que la synthèse considérée se place sous l'autorité de la Règle de son destin, sinon intellectuellement voulu, à tout le moins existentiellement. Sartre le dit en clair :

Dans la mesure où leurs tentatives participent encore de l'esprit de sérieux et où ils peuvent croire encore que leur mission de faire exister l'en-soi-pour-soi est écrite dans les choses, ils sont condamnés au désespoir (L'etre et le néant 721).

Entendons cela ainsi: ils sont encore la proie de la Valeur, de la fameuse synthèse de la condition humaine qui semblait pourtant faire leur originalité par rapport à la synthèse harmonieuse du sensible et de la loi morale chez Kant, c'est-à-dire des motivations particulières et de la liberté pour réaliser leur essence. Mais c'est cette synthèse ou cette essence elle-même qui est suspecte pour Sartre, dans la mesure où les agents moraux respectifs n'assument pas la découverte que chacun est l'être par qui les valeurs existent. C'est alors ainsi que la liberté prendra conscience d'elle-même et se découvrira dans l'angoisse comme l'unique source de la valeur (L'etre et le néant 722), ou encore dans la mesure où l'équivalence d'une option ou de l'autre, au regard de la conscience de soi qui en est prise, ne semble pas revendiquer cette liberté dans et à travers l'option adoptée, mais bien plutôt le contenu du but à atteindre comme objectif ou terminus ad quem.

C'est ici que survient le paradoxe ou l'aporie de l'ambition sartrienne de la liberté : comment échapper dans l'action à cette loi de l'Essence réalisant l'en-soi-pour-soi si toute action est à la fois but idéalement visé et moyen matériellement extrait de la glèbe des choses et du corps ? Trouve-t-on des héros sartriens à la hauteur de l'authenticité ? Gœtz est-il un héros dialecticien authentique lorsqu'il dit: «Il y a cette guerre à faire et je la ferai ! » (Théâtre complet 510) prenant la tête des paysans insurgés ? Il semble bien qu'il se défausse ou se décharge de sa liberté en s'inscrivant sous l'autorité de la situation. Effectivement, dirions-nous, sauf si l'on s'aperçoit que l'itinéraire parcouru par Gœtz au cours 
de la pièce nous donne une garantie du caractère non aliéné de son engagement. Ayant brûlé tous ses vaisseaux, il n'a plus que sa liberté et celle des autres en situation comme justification de son action: il l'a conquise en tuant le prêtre du bas clergé, Heinrich, au demeurant traître au peuple, donc mis un terme à toute loi n'ayant plus que celle de sa liberté pour s'orienter dans l'auto-affirmation d'elle-même.

On comprendra sans doute mieux le problème par la difficulté à faire la part des choses vis-à-vis du scepticisme fatigué et relativiste des demis habiles. On finit par renvoyer dos-à-dos toute option - pour autant qu'il y ait radicale synthèse de la facticité et de la transcendance revendiquée par la liberté respective de chaque attitude - au regard des habiles ; ceux qui dénoncent cet engagement pour l'esprit de sérieux qui y est attaché en tant que la liberté, ou l'activisme, est fétichisé comme moyen de réaliser l'objectif. On comprendra d'autant mieux ce problème par le passage suivant : «à ceux qui croient que l'essence est incontournable et qu'ils en bénéficient comme d'un ange gardien par leur mérite. Mais je leur demanderai d'abord s'ils sont bien sûr d'être eux-mêmes. Cette paix qui règne en eux, sais-je s'ils ne l'ont pas obtenue en faisant leur soumission à un protecteur étranger qui règne à leur place ? Celui que j'entends une fois prononcer ces mots : « Nous médecins ... », je sais qu'il est en esclavage. Ce nous médecins est son moi, créature parasitaire qui lui suce le sang. Et ne fût-il que lui-même, il y a mille façons d'être livré à soi comme aux bêtes, de nourrir avec sa propre chair une idole inflexible et insatiable. Car il n'est permis à personne de dire ces simples mots : je suis moi. Les meilleurs, les plus libres peuvent dire : j'existe. C'est déjà trop » (Saint Genet 85). 


\section{Ouvrages cités}

Deleuze, Gilles. Logique du sens. Paris : Les Editions de minuit, 1982.

Howells, Christina. The Necessity of Freedom. Cambridge University Press : Cambridge, 1988.

Kant, Immanuel. La Religion dans les limites de la simple raison. Paris: Librairie philosophie, 1965.

Pascal, Blaise. Pensées et opuscules. Ed. Léon Brunschvicg. Paris : Hachette, 1967.

Sartre, Jean-Paul. Carnets de la drôle de guerre. Septembre 1939 - mars 1940. Nouvelle édition augmentée d'un carnet inédit. Paris : Gallimard, 1995.

—. L'etre et le néant. Essai d'ontologie phénoménologique. Paris : Gallimard, 1943.

—. Saint Genet. Comédien et martyr. Paris : Gallimard, 1952.

—. Théâtre complet. Paris : Gallimard. Ed. de la Pléiade, 2005. 